\title{
A narrative of the attending nurse model implementation
}

\author{
Terry T Fulmer ${ }^{1}$, Eloise Cathcart ${ }^{2}$, Kimberly K Glassman ${ }^{3}$, Wendy C Budin ${ }^{2,}{ }^{3}$, Madeline A Naegle ${ }^{2}$, \\ Nancy VanDevanter ${ }^{2}$ \\ 1. Northeastern University, Boston, United States. 2. New York University, New York, United States. 3. NYU Langone \\ Medical Center, New York, United States.
}

Correspondence: Terry T Fulmer. Address: Northeastern University, 360 Huntington Avenue Boston, MA 02115, United States. Email: T.fulmer@neu.edu

Received: July 23, 2013

DOI : $10.5430 /$ jnep.v4n3p94
Accepted: September 24, 2013 Online Published: November 3, 2013

URL: http://dx.doi.org/10.5430/jnep.v4n3p94

\section{Abstract}

In this clinical practice exchange, we discuss how faculty at the New York University College of Nursing (NYUCN) and nurse executives at NYU Langone Medical Center (NYULMC) partnered with staff nurses to implement an attending nurse model and further describe how the model facilitates collaboration as well as clinical knowledge development in practicing nurses. The attending nurse model hastens the experiential learning essential for practice development by having faculty and nurse executives provide situated learning, teaching and coaching to advanced beginner and competent practicing nurses. The model supports the fact that learning in the patient care setting is inextricably linked to the delivery of patient care and shows how faculty can contribute to effective outcomes for patients. By modeling professional comportment, helping nurses to put language to salient issues, and coaching nurses about how to participate in interdisciplinary rounds, attending nurses help young clinical staff become active participants in interprofessional dialogue. Nurse executives recognize this model as an innovative and cost efficient way to help clinical staff gain essential knowledge and skill of the practice. For faculty, teaching is enhanced by engagement in actual patient situations which are richer and more complex than theoretical models and serve as a reminder of the centrality of the nurse-patient relationship in both the work of the discipline and the organization.

\section{Key words}

Practice-academe partnerships, Clinical knowledge development, Situated teaching and coaching, Interprofessional collaboration, Practice innovation

\section{Overview}

Nurses entering practice today are immediately called upon to provide care to patients with complex medical and psychosocial needs. Despite the presence of sophisticated nurse residency programs and precepted experiences, new nurses are challenged because they are expected to collaborate effectively with physician colleagues and other members of the healthcare team as a prerequisite for safe and effective patient care ${ }^{[1]}$. In order to communicate effectively about the patient's care needs, the nurse must be able to see what is salient in the patient's situation, be able to put language to her assessment, and have the moral agency to live out her role as advocate and often the "voice" for the patient and family. 
However, the developmental work of new graduate nurses (advanced beginners) is focused on completing tasks within the time demands of the clinical setting and learning to use and adapt rules and theoretical knowledge in concrete situations. Nurses new to practice tend to think analytically and are most comfortable following prescribed procedures. Their performance is mostly normative and mostly safe; they understand moral agency as the responsibility to get the patient through the day and accomplish all the tasks and procedures that are required ${ }^{[2]}$.

Between the second and third year of practice, nurses may be progressing to "competent" and beginning to imagine how it is possible to make a difference in ways that are more significant than solely managing the technical aspects of the patient's care. They are beginning to see that being a nurse is more profound than mastering tasks and getting through the day; that the practice offers the opportunity to make a real difference in the life of another human being. At the same time they are coming face-to-face with the suffering, vulnerability, courage and resilience which are all part of the human condition, issues which were hidden from the advanced beginner who was primarily concerned with getting the patient safely through the day. At the competent stage, nurses realize the limitations of theory and rules in prescribing the correct intervention for a given situation, and see that judgment is critical to assuring good outcomes for the patient ${ }^{[2]}$. This can be a time of great awakening for the nurse as well as a time of great anxiety, and safe passage into this important competent phase of practice development still depends on learning acquired from working alongside more experienced nurses. The competent nurse's inability to see or interpret a salient clinical sign or symptom is corrected by others' experiential wisdom and skill of seeing ${ }^{[3]}$.

At New York University we adapted and enhanced the attending nurse model to help clinical nurses new to practice and those transitioning into the competent level of practice stand with their interdisciplinary colleagues in processes of collaboration ${ }^{[4,5]}$. The model brings senior seasoned NYU College of Nursing faculty and NYULMC nurse executives into the practice experience to participate as attending nurses in interprofessional patient rounds. Attending nurses make links between seeing the most important issues in the situation and describing them to clinical nurses in ways that are clear and enhance learning. The attending nurse, given advanced clinical experience, can assess the situation quickly without going through a time-intensive process of considering alternative solutions and is rapidly able to see what needs to be done for the patient and family ${ }^{[6]}$. This attending sees the ethical demand of practice as authentically engaging with the patient and family to walk with them through the experience of illness, with the intent of restoring health and preserving dignity in whatever way is meaningful for the particular patient ${ }^{[7]}$. In this consulting role, attending nurses offer their own clinical wisdom and expertise to coach staff nurses to participate in the collegial dialogue and decision making processes.

The purpose of this paper is to provide the narrative of the attending model adaptation and implementation at NYULMC and suggest how such models can advance faculty, administrator, and nurse executive engagement in the advancement of clinical nursing practice. The model is built upon our belief that the patient care setting is a place where learning is inextricably linked to the delivery of patient care ${ }^{[8]}$.

\section{Experiential learning}

The spiral of learning from experience described in experimental learning theory can help learners learn how to learn. Kolb has noted, “..by consciously following a recursive cycle of experiencing, reflecting, thinking and acting, [they] can increase their learning power” ${ }^{[9]}$. In our case, staff nurses who experience attending nurse leadership can reflect on what they have observed, make meaning of the experience, and bring it into their own practice. Others have documented the impact on experiential learning in medical school education, using quality improvement projects as experiential engagement ${ }^{[10]}$.

The accrual of experiential learning is essential for a nurse to achieve expertise in practice. Experiential learning occurs best when the nurse (or any clinician) approaches new clinical situations with focused attentiveness and openness to having preconceived notions turned around in patient care settings that are safe and supportive learning environments ${ }^{[11]}$. 
Learning the skilled knowledge of the practice requires tolerance for error, so a healthy work environment characterized by respect and civility which is supportive to young practitioners is a prerequisite for learning how to be an excellent nurse ${ }^{[12,13]}$. The challenge for nurse managers is how to connect nurses and patients in relationships that ensure the best care for patients and maximum experiential learning for the nurse while protecting the safety of both ${ }^{[11]}$.

\section{Attending nurse model}

The attending nurse model and qualitative outcomes from the model have been described elsewhere ${ }^{[4,5]}$. The guiding principle of the model is that senior, seasoned nurses, (here we emphasize those in academic and executive roles) should have a regular presence and an impact in the nursing practice learning environment. Like attending physicians, the nurse attending model provides the ongoing opportunity for nursing faculty and nurse executives to stay current with the demands of clinical practice, literature related to clinical conditions, and advancements in safety, quality and technology. Staff nurses benefit from the opportunity to observe attending nurses as peers with attending physicians as patient care plans are developed. Attending nurses help accelerate evidence-driven practice plans by assisting the staff nurses with literature reviews, evidence-based practice protocols and current health policy related to practice.

\section{I mplementing the model}

The Chief Nursing officer of the NYU Langone Medical Center and the Dean of the NYU College of Nursing joined together to commit to integrating senior nursing faculty and nurse executives into the clinical practice of staff nurses at the medical center in order to bring to bear the clinical scholarship and experience to the staff. In addition, the staff could alert the nurse attendings to the ongoing myriad of changes that take place in the practice environment daily. The two nurse leaders shared a commitment to nurturing and supporting nursing practice development and worked together to advance the attending nurse model, piloted earlier at the Mount Sinai Medical Center in New York City ${ }^{[4]}$. They invited nurse executives at NYULMC and nursing faculty from the NYU College of Nursing to serve as attending nurses, and participate in daily patient rounds, each for a two-week block. The inclusion of nurse executives was a new element of the model, because prior, only college faculty served as attendings. Here, the senior nursing executives from NYULMC joined as attendings, to bring their clinical expertise and benefit from the opportunity to partner with staff nurses in practice. Attending nurses role modeled for less experienced nurses how to bring the nursing perspective into rounds, and facilitate greater nurse participation by coaching clinical nurses to offer their observations about the patient's situation and advocate for patient and family needs.

Two medical-surgical units were selected as these units had a preponderance of nurses whose experience ranged from being new to practice to three years. Through several meetings with the lead physician and nurse manager (one nurse manager was overseeing both units due to a nurse manager vacancy), the CNO and the Dean explained the goals of the attending nurse model and obtained support for its introduction ${ }^{[5]}$. They also engaged the nurse manager, the coordinator of NYU's graduate program in nursing administration and the lead hospitalist to oversee the model's further development and implementation.

The nurse manager and graduate program coordinator met with the physician overseeing patient rounds to discuss the rounding process. The physician expressed support for involving nurses in rounds and recognized the potential usefulness of having a senior nurse present to support and encourage nurse participation. The physician also noted that four different medical teams rounded on the units each day and proposed working with one team to introduce the attending nurse model.

Nurse executives and nursing faculty who had a sound understanding of nursing practice environment and a facility for coaching clinical staff were invited to participate in the attending nurse role. The graduate program coordinator met with each person who agreed to take on the attending nurse role to further explain the model's goals and how it would be implemented. She also developed a schedule for the first year, assigning herself to the first two-week rotation as an attending nurse. 
The graduate coordinator for the nursing administration academic program worked with the attending nurse and the nurse manager, and introduced the attending nurse model to clinical nurses on 17E and $17 \mathrm{~W}$. The two units comprise 70 beds and care for an acute population of medical-surgical patients. The average length of stay is approximately five days, but many patients have chronic conditions and return periodically for care and are well known to the nursing staff. For the nurse manager, finding ways to support these less experienced nurses and helping them develop their practice was an ongoing priority. Other priorities included fostering a collaborative environment, where nurses, physicians, and other members of the care team work together to meet each patient's needs. She believed the attending nurse model would be useful in helping achieve both goals.

Through staff meetings and one-on-one discussions with clinical nurses, the nurse manager explained the goals of the attending nurse model and how it would work day to day. As part of these discussions, she emphasized the importance of having the nursing perspective inform the plan of care and set an expectation that all nurses participate in rounds on their patients. In response, the clinical nurses expressed interest in joining rounds and for having an expert, senior nurse in attendance. However, they also expressed concern about the time commitment and inconvenient timing of rounds, noting they began at 9 a.m., one-half hour after nursing report was over and before nurses had a chance to fully assess their patients. Recognizing nurses' time constraints, the nurse manager tapped the units' charge nurses, asking them to support nurse participation in rounds by covering patients when necessary. An additional part of the implementation process included conducting focus groups with clinical nurses to obtain a preliminary sense of an evaluation plan for the attending nurse model ${ }^{[5]}$.

\section{First attending nurse rotation}

The graduate program coordinator joined rounds as the first attending nurse in August of 2009. In advance, the nurse manager introduced her to staff, giving her an opportunity to learn more about the nurses and introduce herself and her nursing background. Initially, the attending nurse simply observed how clinical nurses participated, noting that in many cases nurses were unable to attend rounds. When nurses were present, their contributions were often limited to reporting vital signs and other data or reconciling problems with physician orders.

As attending nurses became more familiar with patients and the rounding routine, they began sharing their own nursing observations. They also began taking a few minutes before a new patient was presented to speak with the patient's nurse and discuss the nurse's questions and concerns regarding the treatment plan. Through these check-ins, the attending nurses validated clinical nurses' judgments about their patients and encouraged them to share their observations in rounds. Additionally, during rounds they made a point of acknowledging clinical nurses by name and inviting them to share their observations and concerns with the team.

Like the attending physician, the attending nurse also played a role in teaching and role modeling professional comportment. In one instance, for example, a member of the house staff presented a patient who had been non-compliant with her diabetic care plan, and neglected to note that the patient had been blind since an early age. Upon meeting the patient, the resident did not introduce himself and proceeded to conduct a perfunctory physical assessment. When the clinicians reconvened in the hallway, the attending nurse shared her observation that meeting and talking with patients always made a difference in deepening her understanding of how particular patients perceive and cope with their illness. In this case it helped her appreciate what it might have meant for this patient to live with a condition like blindness, and how that might affect her ability to comply with a medical regimen. This seemingly simple observation helped to remind the team of this patient's unique situation and shifted the tenor of the discussion from one of blaming the patient to one more focused on supporting and helping her manage her disease.

A new attending nurse joined rounds every two weeks as it was a two week rotation. Each time, the nurse manager facilitated the attending nurses' transition into the role by introducing her to the clinical nurses and physicians on the unit. The nurse manager also continually reinforced with nurses the value of patient rounds and helped troubleshoot problems 
that hindered nurse participation. For example, she asked charge nurses to give staff advance notice of when their patients would be discussed so that nurses could factor rounds into their morning routine. She also worked with the attending physicians to make rounds more predictable by asking them to discuss patients in a set order whenever possible so that nurses could anticipate their patient's place in the rounding queue. As time went on, nurse participation in rounds and the attending nurse model gradually became part of the daily routine.

\section{Reactions to the model}

After one year, all of the attending nurses had completed at least one two-week rotation, and many had completed two; an attending nurse had been present at rounds every week-day throughout the year. Most of the nurses filling the attending nurse role found it both challenging and rewarding as expressed in this comment: "My experience as an attending nurse helped me remember what clinical nurses do, what their daily experience is and how I can help them advance their practice." Faculty serving in the role described how the experience enriched their teaching by providing them with actual patient situations which are always richer than theoretical models, reminding them of the centrality of the nurse-patient relationship, and helping them gain a renewed appreciation of the complexity of the patient care process and the demands placed on clinical nurses and nurse managers. When it came time to develop an attending nurse schedule for the second year, approximately eighty percent of the original attending nurses volunteered to serve in the role again. This is a testament to the commitment of the attendings to the support of practicing nurses.

Clinical nurses also expressed appreciation for the attending nurse role and noted how nurse-physician communication had improved since patient rounds and the attending nurse model had been introduced ${ }^{[5]}$. In addition to finding it easier to plan their patients' care, they often needed to make fewer phone calls to physicians during the day, since many of their questions were addressed in morning rounds. Perhaps most importantly, the nurses said they believed their voices were being heard and that their concerns were reflected in the plans of care formulated for their patients. Despite some lingering concerns about the time required for rounding, they vocalized a desire to continue rounds with attending nurses and the interdis-

ciplinary team.

The nurse manager observed a marked increase in the number of nurses joining rounds each day and a perception on her part and that of the staff that coordination of care has improved. Patients are now receiving more accurate, timely and clearer information, reinforcing for them that their care is being coordinated by the team. While the time spent in rounds was still a challenge, most nurses joined at least part of the discussion on their patients. The nurse manager also noted that nurse participation in rounds seemed to have a beneficial effect on interprofessional collaboration and communication outside of rounds. In the past, house staff often decided treatment plans for patients and entered orders without discussing them with nurses. As the number of nurses joining rounds steadily increased, house staff more frequently introduced themselves to clinical nurses and consulted with them before changing a patient's treatment plan. They also began consulting with nurses before admitting a patient to the floor to confirm that the nurses could manage another admission.

Additionally, the nurse manager received positive feedback from many clinical nurses about the attending nurse role. They appreciated the encouragement and coaching they received from attending nurses, and the clinical nurses expressed pride at having a nurse co-equal to the attending physician participate in rounds. The nurse manager and graduate program coordinator noted that one of their most rewarding experiences involved a nurse who had previously been hesitant to speak up in rounds. When it came time to discuss her patient, the nurse calmly responded to the team's questions before adding, "Here's what I think is going on..."

\section{Discussion}

Nurse leaders in hospital settings are continually challenged to find ways to support the experiential learning which is essential for practice development. Having clinical experts available to provide situated clinical teaching is especially 
important for nurses at the advanced beginner and competent levels of practice ${ }^{[3]}$. Organizations often turn to nurse preceptors and clinical nurse specialists (CNSs) to provide much of this teaching and to share experiential learning and clinical wisdom. However, the decline in CNS programs in graduate nursing education and the corresponding move from the CNS role to that of nurse practitioner, has led many nurse executives to search for alternative resources to help staff gain the essential knowledge and skill of the practice.

The attending nurse model is an exemplar of how existing expert nursing resources can be leveraged to help clinical staff learn and practice skills required for effective patient care. By role modeling professional comportment, teaching nurses to identify and put language to salient issues, and coaching and encouraging nurse participation in rounds, attending nurses help clinical staff become active participants, rather than passive observers, in the interprofessional dialogue and care planning that takes place in rounds. As participants, nurses learn and practice skills required for the deliberate and collaborative planning integral to achieving competent practice ${ }^{[14]}$.

Feedback from the staff nurses, observations by the nurse manager, and comments offered by attending physicians suggest that the attending nurses facilitated nursing involvement in patient rounds and were instrumental to helping nurses gain the confidence and skills needed to share their observations. As noted by the nurse manager, the enhanced nurse participation in rounds also had a positive effect by leading to improved communication and collaboration between nurses and physicians outside of rounds and contributing to a more collaborative culture on the unit.

In our view, several factors were instrumental to the successful implementation of the attending nurse model at NYULMC. Key among these was the partnership developed and modeled by the CNO and Dean, their leadership in obtaining support from physician colleagues, and their ongoing support for implementation efforts. Physician support for the model and for interprofessional participation in rounds was also critical. The lead physician on 17E and 17W, in particular, not only welcomed the participation of nurses in rounds, but also role modeled and set expectations for interprofessional collaboration among house staff on the units.

Having one nurse from academe and another from the practice setting co-lead the model's implementation was also important. The coordinator of the graduate program in nursing administration was well positioned to identify faculty interested in filling the attending nurse role and develop a rotation schedule that did not conflict with other responsibilities. Additionally, by serving as an attending nurse and completing the first two-week rotation, she was able to identify and help trouble-shoot logistical challenges and counsel other attending nurses about the role. Similarly, the nurse manager was uniquely positioned to create an environment supportive of the model's introduction. By continually emphasizing the importance of participating in rounds, explaining the attending nurse model to staff, and helping nurses factor rounds into their workflow, the nurse manager engendered interest, enthusiasm, and support for the model among clinical nurses. She was also able to build on her collegial relationship with physicians to obtain their support for nurse participation in rounds and the attending nurse role.

Nurses are more likely to participate in rounds if they can anticipate when their patients will be discussed and plan accordingly. In the future, we will be working with the attending physicians to bring increased standardization to the rounding process as medical rounds are revised. In addition to participating in rounds, we will also work solely with nurses in unit-based meetings to review interesting patient cases as a method of enhancing nurses' clinical knowledge and preparing them for interdisciplinary conversation.

Now that a model of interdisciplinary communication and collaboration has been established, efforts will also be geared toward developing and articulating the clinical knowledge, judgment and moral agency of these new to practice and competent nurses through case studies, evidence-based problem solving and reflection on experiential learning. With these changes, we believe the attending nurse model will have an even greater impact on nursing practice development and ultimately, the quality and safety of patient care. 


\section{References}

[1] Edmondson, \& A. Teaming: How Organizations Learn, Innovate and Compete in the Knowledge Economy. San Francisco, CA: John Wiley and Sons, 2012.

[2] Benner, P., Tanner, C., \& Chesla, K. Expertise in Nursing Practice: Caring, Clinical Judgment and Ethics. New York: Springer, 2009.

[3] Benner, P., Sutphen, M., Leonard, V., \& Day, L. Educating Nurses: A Call for Radical Transformation. San Francisco: Jossey-Bass, 2010.

[4] Fulmer, T. The Attending Model in Nursing. Applied Nursing Research. 2010; 13(3): 113-114. PMid:10960994 http://dx.doi.org/10.1053/apnr.2000.0130113

[5] Fulmer, T., Cathcart, E. B., Glassman, K., Budin, W., Naegle, M., \& Van Devanter, N. The Attending Nurse: An Evolving Model for Integrating Nursing Education and Practice. The Open Nursing Journal, 2011. PMid:21660179 http://dx.doi.org/10.2174/1874434601105010009

[6] Cathcart, E. B., \& Greenspan, M. (in press). The Role of Practical Wisdom in Nurse Manager Practice: Why Experience Matters. Journal of Nursing Management

[7] Cathcart, E. B., \& Greenspan, M. A New Window into Nurse Manager Development: Teaching for the Practice. Journal of Nursing Administration. 2012; 42(12): 557-561. PMid:23151927 http://dx.doi.org/10.1097/NNA.0b013e318274b52d

[8] Cathcart, E. B., \& Fillipon, K. G. Strengthening the Practice of Nursing. In M. Hickey \& P. B. Kritek (Eds.), Change Leadership in Nursing: How Change Occurs in a Complex Hospital System. New York: Springer, 2011.

[9] Kolb, A., \& Kolb, D. The Learning Way: Meta-cognitive Aspects of Experiential Learning. Simulation \& Gaming. 2009; 40(3): 297-327. http://dx.doi.org/10.1177/1046878108325713

[10] Ogrinc, G., Nierenberg, D., \& Batalden, P. Building Experiental Learning about Quality Improvement into a Medical School Curriculum: The Dartmouth Experience. Health Affairs. 2011; 30(4): 716-722. PMid:21471493 http://dx.doi.org/10.1377/hlthaff.2011.0072

[11] Cathcart, E. B. The Role of the Chief Nursing Officer in Leading the Practice: Lessons from the Benner Tradition. Nursing Administration Quarterly. 2008; 32(2): 87-91. PMid:18360204 http://dx.doi.org/10.1097/01.NAQ.0000314536.91122.8b

[12] Shirey, M. Authentic Leaders Creating Healthy Work Environments for Nursing Practice. American Journal of Critical Care. 2006; 15(3): 256-267. PMid:16632768

[13] Smith, L. M., \& al., e. Effects of Workplace Incivility and Empowerment on Newly Graduated Nurses' Organization Committment. Journal of Nursing Management. 2010; 18: 1004-1015. PMid:21073572 http://dx.doi.org/10.1111/j.1365-2834.2010.01165.x

[14] Cathcart, E. B., Greenspan, M., \& Quin, M. The Making of a Nursing Manager: The Role of Experiental Learning in Leadership Development. Journal of Nursing Management. 2010; 18(4): 440-447. PMid:20609048 http://dx.doi.org/10.1111/j.1365-2834.2010.01082.x 